\title{
Genuine phase diffusion of a Bose-Einstein condensate in the microcanonical ensemble: A classical field study
}

\author{
A. Sinatra and Y. Castin \\ Laboratoire Kastler Brossel, Ecole Normale Supérieure, \\ UPMC and CNRS, 24 rue Lhomond, 75231 Paris Cedex 05, France
}

\begin{abstract}
Within the classical field model, we find that the phase of a Bose-Einstein condensate undergoes a true diffusive motion in the microcanonical ensemble, the variance of the condensate phase change between time zero and time $t$ growing linearly in $t$. The phase diffusion coefficient obeys a simple scaling law in the double thermodynamic and Bogoliubov limit. We construct an approximate calculation of the diffusion coefficient, in fair agreement with the numerical results over the considered temperature range, and we extend this approximate calculation to the quantum field.
\end{abstract}

PACS numbers: 03.75.Kk, 03.75.Pp

\section{INTRODUCTION}

Phase coherence is one of the most prominent properties of Bose-Einstein condensates, relevant for applications of condensates in metrology and quantum information 1]. The issue of condensate phase dynamics and phase spreading at zero temperature due to interactions has been extensively studied in theory [2] and experiments $[3,4,5]$. There is a renewed interest in this issue of temporal phase coherence due to the recent studies in low dimensional quasi condensates, both experimentally [6, 7, 8] and theoretically [9]. The present work addresses the problem of the determination of fundamental limits of phase coherence in a true three dimensional Bose-Einstein condensates at non zero temperature.

The effect of finite temperature on phase coherence in a Josephson junction realized by a condensate trapped in a double well potential has been studied in [10] and [11]. The situation is different when the two condensates are separated. In this case there is no restoring force for the relative phase which then evolves independently in the two BECs 12]. The effect of the temperature in this case, joint to the effect of the interactions, is to provide a spreading in time of the relative phase.

In a previous work [13], we considered a condensate prepared in an equilibrium state in the canonical ensemble. In that case we could show using ergodicity that the phase change of the condensate during a time $t$ has a variance which grows proportionally to $t^{2}$. In other words, the condensate phase spreading in the canonical ensemble is ballistic [14] and not diffusive [15, 16, 17, 18]. As we could calculate in [13] using an ergodic theory, the coefficient of this super-diffusive thermal spreading is proportional to the variance of the energy in the considered equilibrium state. If we now suppress the fluctuations of energy in the initial state, by moving from the canonical ensemble to the microcanonical ensemble, the ballistic thermal spreading disappears and one may expect that the condensate phase undergoes a genuine diffusion in time. In the present work we show that this is indeed the case and we study this genuine phase diffusion numerically within the classical field model [19, 20, 21, 22, 23, 24] described in section [I. The numerical results are presented in section [II] and their analysis shows the existence of simple scaling laws and of an universal curve giving the phase diffusion coefficient in the double thermodynamical limit $(N \rightarrow \infty$, volume $V \rightarrow \infty$ density $\rho=$ constant $)$ and Bogoliubov limit $(N \rightarrow \infty$, coupling constant $g \rightarrow 0, N g=$ constant). In section IV we derive an aproximate formula for the diffusion coefficient, that we compare to the numerical results and that we also extend to the quantum field. We conclude in section $\mathrm{V}$.

\section{CLASSICAL FIELD MODEL AND NUMERICAL PROCEDURE}

We consider a lattice model for a classical field $\psi(\mathbf{r})$ in three dimensions. The lattice spacings are $l_{1}, l_{2}, l_{3}$ along the three directions of space and $d V=l_{1} l_{2} l_{3}$ is the volume of the unit cell in the lattice. We enclose the atomic field in a spatial box of sizes $L_{1}, L_{2}, L_{3}$ and volume $V=L_{1} L_{2} L_{3}$, with periodic boundary conditions. To guarantee efficient ergodicity in the system we choose non commensurable square lengths in the ratio $L_{1}^{2}: L_{2}^{2}$ : $L_{3}^{2}=\sqrt{2}:(1+\sqrt{5}) / 2: \sqrt{3}$. The lattice spacings squared $l_{1}^{2}, l_{2}^{2}, l_{3}^{2}$ are in the same ratio.

The field $\psi$ may be expanded over the plane waves

$$
\psi(\mathbf{r})=\sum_{\mathbf{k}} a_{\mathbf{k}} \frac{e^{i \mathbf{k} \cdot \mathbf{r}}}{\sqrt{V}}
$$

where $\mathbf{k}$ is restricted to the first Brillouin zone, $k_{\alpha} \in$ $\left[-\pi / l_{\alpha}, \pi / l_{\alpha}[\right.$ and $\alpha$ labels the directions of space.

We assume that, in the real physical system, the total number of atoms is fixed, equal to $N$. In the classical field model, this fixes the norm squared of the field:

$$
d V \sum_{\mathbf{r}}|\psi(\mathbf{r})|^{2}=N
$$

Equivalently the density of the system

$$
\rho=\frac{N}{V}
$$


is fixed for each realization of the field. The evolution of the field is governed by the Hamiltonian

$$
H=\sum_{\mathbf{k}} \tilde{E}_{k} a_{\mathbf{k}}^{*} a_{\mathbf{k}}+\frac{g}{2} \sum_{\mathbf{r}} d V \psi^{*}(\mathbf{r}) \psi^{*}(\mathbf{r}) \psi(\mathbf{r}) \psi(\mathbf{r}),
$$

where $\tilde{E}_{k}$ is the dispersion relation of the non-interacting waves, and the binary interaction between particles in the real gas is reflected in the classical field model by a field self-interaction with a coupling constant

$$
g=\frac{4 \pi \hbar^{2} a}{m}
$$

where $a$ is the $s$-wave scattering length of two atoms.

As a matter of a fact we use here the same refinement as in [13] consisting in modifying the dispersion relation in order to obtain for the ideal gas the correct quantum values of the mean occupation numbers at equipartition

$$
\frac{1}{e^{\beta \hbar^{2} k^{2} / 2 m}-1}=\frac{k_{B} T}{\tilde{E}_{k}} .
$$

However we do not expect this to have a large impact here as we put a cut-off at an energy of the order of $k_{B} T$. More precisely we choose the number of the lattice points in a temperature dependent way, such that the maximal Bogoliubov energy on the lattice is equal to $k_{B} T$ :

$$
\max _{\mathbf{k}}\left[\left(\hbar^{2} k^{2} / 2 m\right)\left(2 \rho g+\hbar^{2} k^{2} / 2 m\right)\right]^{1 / 2}=k_{B} T .
$$

The discretized field has the following Poisson brackets

$$
i \hbar\left\{\psi\left(\mathbf{r}_{1}\right), \psi^{*}\left(\mathbf{r}_{2}\right)\right\}=\frac{\delta_{\mathbf{r}_{1}, \mathbf{r}_{2}}}{d V}
$$

where the Poisson brackets are such that $d f / d t=\{f, H\}$ for a time-independent functional $f$ of the field $\psi$. The field then evolves according to the non linear equation [25]

$$
i \hbar \partial_{t} \psi=\left\{k_{B} T\left[\exp \left(-\beta \frac{\hbar^{2}}{2 m} \Delta\right)-1\right]+g|\psi(\mathbf{r}, t)|^{2}\right\} \psi .
$$

We introduce the density and the phase of the condensate mode

$$
a_{0}=e^{i \theta} \sqrt{N_{0}}
$$

The quantity of interest is the variance of the condensate phase change during $t$ :

$$
\operatorname{Var} \varphi(t)=\left\langle\varphi(t)^{2}\right\rangle-\langle\varphi(t)\rangle^{2}
$$

where

$$
\varphi(t)=\theta(t)-\theta(0) .
$$

The averages are taken over stochastic realizations of the classical field, as the initial field samples the microcanonical ensemble with an energy $E$. For convenience, we parametrize the microcanonical ensemble by the temperature $T$ such that the mean energy of the field in the canonical ensemble at temperature $T$ is equal to $E$.

To generate the stochastic initial values of the classical field we proceed as follows. (i) First we generate 1000 stochastic fields in the canonical ensemble at temperature $T$, as explained in [13], and we compute the average energy of the field $\langle E\rangle_{\text {can }}$ and its root mean squared fluctuations $\sigma=\sqrt{\operatorname{Var} E}$. (ii) We generate other fields, still in the canonical ensemble, and we filter them keeping only realizations with an energy $E$ such that $\left|E-\langle E\rangle_{\text {can }}\right| \leq 0.01 \sigma / 2$. (iii) We let each field evolve for some time interval with the Eq.(9) to eliminate transients due to the fact that the Bogoliubov approximation, used in the sampling, does not produce an exactly stationary distribution. After this 'thermalization' period we start calculating the relevant observables, as $\psi$ evolves with the same equation (9). In practice this equation is integrated numerically with the FFT splitting technique. The ensemble of data reported here has required a CPU time of about two years on Intel Xeon Quad Core $3 \mathrm{GHz}$ processors.

\section{NUMERICAL RESULTS AND SCALING LAWS}

The first important result that we obtain is the diffusive behavior of the condensate phase. In Fig 1 we show an example of numerical data. From bottom to top, five values of $k_{B} T / \rho g$ are presented for a constant number of atoms $N=2.36 \times 10^{6}$. The wavy line with error bars is the phase variance as a function of time obtained with about 1200 stochastic realizations [26]. The solid line is a linear fit from which we deduce the value of the diffusion coefficient,

$$
\operatorname{Var} \varphi(t) \stackrel{t \rightarrow \infty}{\sim} 2 D t
$$

The diffusive behavior of the condensate phase is strictly related to the long time behavior of the time correlation function $\mathcal{C}$ of the condensate phase derivative $\dot{\varphi}$,

$$
\mathcal{C}\left(\left|t^{\prime}-t^{\prime \prime}\right|\right)=\left\langle\dot{\varphi}\left(t^{\prime}\right) \dot{\varphi}\left(t^{\prime \prime}\right)\right\rangle-\left\langle\dot{\varphi}\left(t^{\prime}\right)\right\rangle\left\langle\dot{\varphi}\left(t^{\prime \prime}\right)\right\rangle,
$$

where we used the fact that $\mathcal{C}$ depends only on $\left|t^{\prime}-t^{\prime \prime}\right|$ for a steady state classical field. By writing $\varphi(t)$ in terms of its time derivative, one obtains [13]

$$
\operatorname{Var} \varphi(t)=2 t \int_{0}^{t} d \tau \mathcal{C}(\tau)-2 \int_{0}^{t} d \tau \tau \mathcal{C}(\tau)
$$

If $\mathcal{C}(t)$ has a non-zero limit at long times, as it was the case in the canonical ensemble [13], $\operatorname{Var} \varphi$ grows quadratically in time. Here, in the microcanonical ensemble $\operatorname{Var} \varphi$ grows linearly in time and we expect that $\mathcal{C}(t) \rightarrow 0$ when $t \rightarrow \infty$. An illustration of that, for two values of the temperature, is given in Fig 2 where, for convenience, 


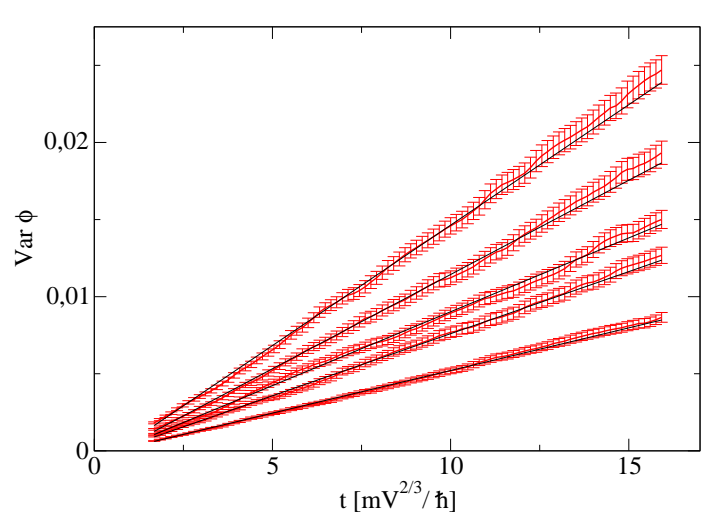

FIG. 1: Variance of the condensate phase change $\varphi(t)$ as a function of time. Wavy line with error bars: Numerical results. Solid lines: A linear fit. From bottom to top, the reduced temperature $k_{B} T / \rho g$ is $9.7,13.2,15.7,19.6,24.2$. The number of atoms is fixed to $N=2.37 \times 10^{6}$. The high energy cut-off is fixed according to (7), on a grid $32^{3}$, so that the temperature slightly varies, from bottom to top: $k_{B} T /\left(\hbar^{2} / m V^{2 / 3}\right)=16864,16411,16212,16010,15854$. The time is in units of $m V^{2 / 3} / \hbar$.

$\mathcal{C}(t)$ is calculated with a simplified formula for the phase derivative [27]

$$
\hbar \dot{\varphi} \simeq-\rho g-\frac{g}{V} \sum_{\mathbf{k} \neq \mathbf{0}}\left(\tilde{U}_{k}+\tilde{V}_{k}\right)^{2}\left|b_{\mathbf{k}}\right|^{2} .
$$

In (16) the $b_{\mathbf{k}}$ are the field amplitudes on the Bogoliubov modes [28].

We now investigate numerically how the diffusion coefficient scales in different limits. First we consider the "Bogoliubov limit" introduced in [29]

$$
N \rightarrow \infty, \quad g \rightarrow 0 \text { with } N g=\text { constant }
$$

the other parameters $\left(V, l_{1}, l_{2}, l_{3}, T\right)$ being fixed [30]. In this limit, the number of non condensed particles converges to a non zero value while the non condensed fraction vanishes. The time evolution of the Bogoliubov occupation numbers $n_{\mathbf{k}}=\left|b_{\mathbf{k}}\right|^{2}$ is then mainly due to terms in the interaction Hamiltonian which are cubic in the non condensed field amplitude and linear in the condensate amplitude and thus of order $\epsilon=g \sqrt{N}$. Physically these cubic terms describe interactions among Bogoliubov modes such as Landau and Beliaev processes [31, 32, 33, 34, which are included in the classical field model [19, 20, 22, 23, 35, 36, 37, 38, 39, 41]. They lead to evolution rates of the $n_{\mathbf{k}}$ of order $\epsilon^{2}$. We thus expect a phase diffusion coefficient of the same order $\epsilon^{2}$, which is $\propto 1 / N$ according to (17). This expectation is confirmed numerically as we show in Fig 3 where we find that $D N$ is constant within the error bars over a factor 5 variation of $N$ and for three considered temperatures.

We now investigate the existence of a thermodynamical limit for the quantity $D N$, given that the Bogoliubov limit is already reached. The thermodynamical limit is
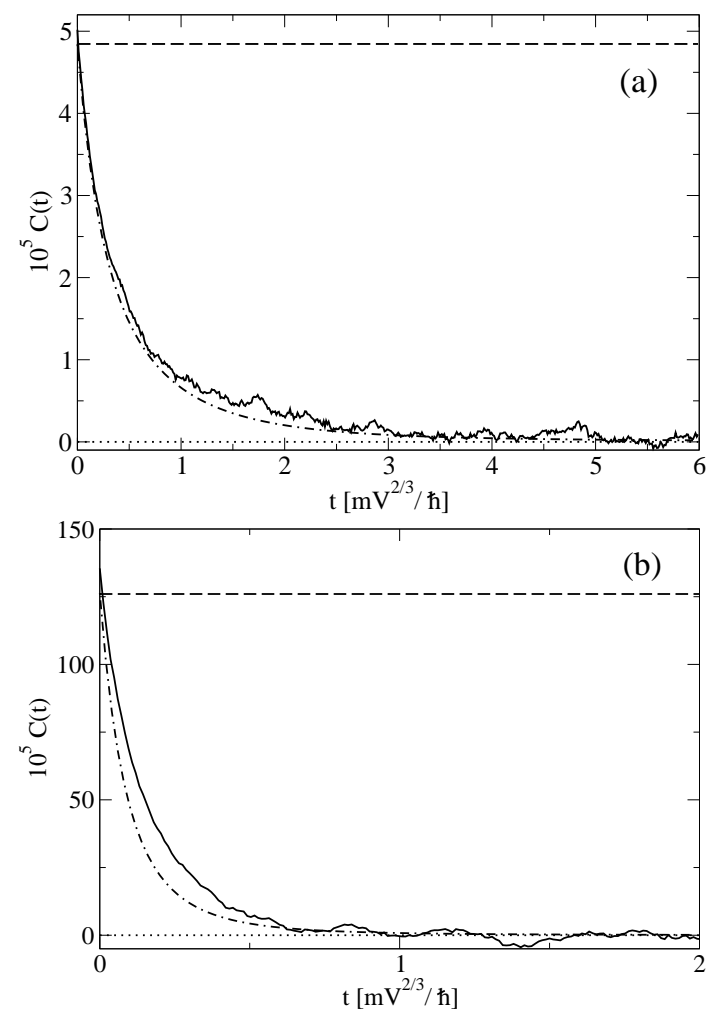

FIG. 2: Correlation function $\mathcal{C}(t)$ of the phase derivative $\dot{\varphi}(t)$ given by the non-oscillating approximation (16), as a function of time. Solid line: Numerical results. Dashed line: Result of Bogoliubov theory. Dashed-dotted line: Prediction of the projected Gaussian approach of section IV The number of atoms is fixed to $N=5 \times 10^{6}$, and the Gross-Pitaveskii chemical potential is fixed to $\rho g=700 \hbar^{2} / m V^{2 / 3}$. In (a) the temperature is $k_{B} T /\left(\hbar^{2} / m V^{2 / 3}\right)=5469$, with a grid size $18^{3}$. In (b) the temperature is $k_{B} T /\left(\hbar^{2} / m V^{2 / 3}\right)=14054$, with a grid size $30^{3}$.

defined as usual as

$$
N \rightarrow \infty, \quad V \rightarrow \infty \text { with } \rho=\text { constant }
$$

the other parameters $\left(g, l_{1}, l_{2}, l_{3}, T\right)$ being fixed. The result is shown in Fig 4 where $D N$ is constant within the error bars, over a factor 5 of variation of $N$ and 4 considered temperatures.

In what follows, using dimensional analysis, we show that for our cut-off procedure (77), the dimensionless quantity $\hbar D N / \rho g$ is a function of a single parameter $k_{B} T / \rho g$ once the Bogoliubov and thermodynamical limits are reached. Six independent physical quantities are present in the model

$$
\left\{\hbar, m, g, V, k_{B} T, N\right\} .
$$

The lattice spacings $l_{1}, l_{2}, l_{3}$ are not independent parameters since their ratios are fixed and their value is determined by (77) once the quantities (19) are fixed. Equivalently we can replace $g$ by $\rho g$ and the volume $V$ by $k_{B} T_{c}$, where $T_{c}$ is the transition temperature of the ideal gas 


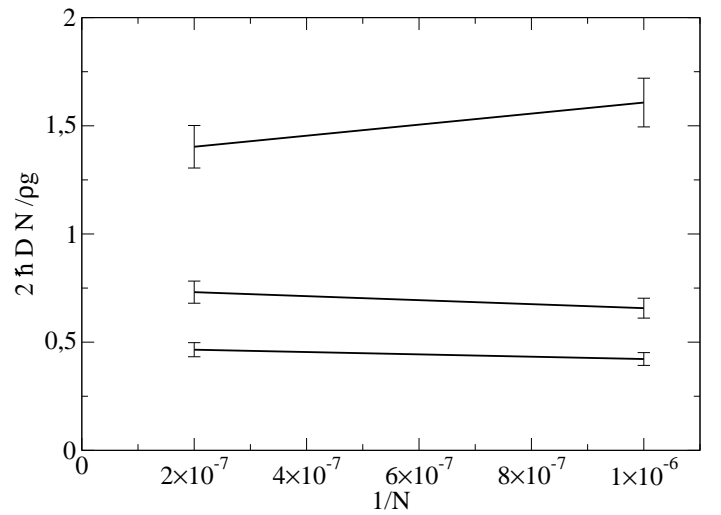

FIG. 3: Scaling of the phase diffusion coefficient in the Bogoliubov limit (17), for a factor 5 variation of the atom number $N$. The Gross-Pitaevskii chemical potential is fixed to $\rho g=700 \hbar^{2} / m V^{2 / 3}$. Points with error bars: Simulation results. The lines connect the points with the same temperature. From bottom to top: $k_{B} T=5469 \hbar^{2} / m V^{2 / 3}$ with a grid size $18^{3}, k_{B} T=6606 \hbar^{2} / m V^{2 / 3}$ with a grid size $20^{3}$, $k_{B} T=9231 \hbar^{2} / m V^{2 / 3}$ with a grid size $24^{3}$.

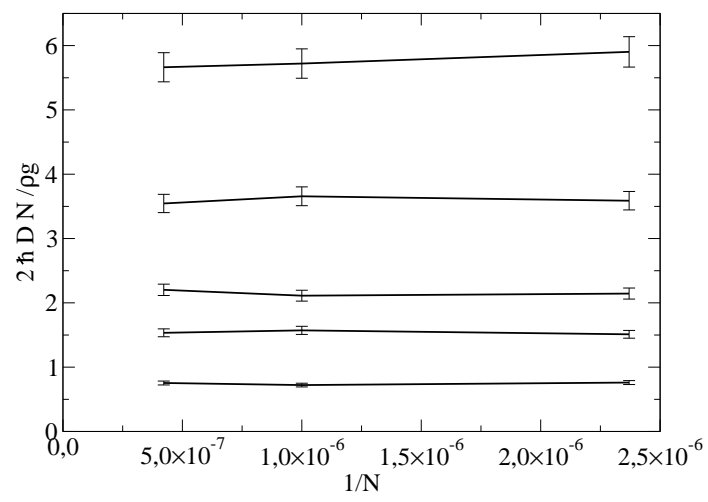

FIG. 4: Scaling of the phase diffusion coefficient in the thermodynamic limit (18), for a factor 5 variation of the atom number $N$. Points with error bars: Simulation results. The lines connect the points with the same $k_{B} T / \rho g$. The points most on the left of the figure, with $N=2.37 \times 10^{6}$, are the ones of Fig[1] with a grid size $32^{3}$. The other points are for a grid size $24^{3}\left(N=10^{6}\right)$ and for a grid size $18^{3}\left(N=4.22 \times 10^{5}\right)$.

given by

$$
\rho\left(\frac{2 \pi \hbar^{2}}{m k_{B} T_{c}}\right)^{3 / 2}=\zeta(3 / 2) .
$$

We then have

$$
\frac{\hbar D N}{\rho g}=f\left(\hbar, m, \rho g, \frac{k_{B} T_{c}}{\rho g}, \frac{k_{B} T}{\rho g}, N\right) .
$$

The three quantities $\hbar, m, \rho g$ can be recombined to form a length, a time and a mass which are three independent dimensioned quantities. Since $f$ and its other three variables are dimensionless, $f$ does not depend of its first three variables. In the thermodynamical limit $N \rightarrow \infty$ so the sixth variable of $f$ drops out of the problem. In the Bogoliubov limit, $k_{B} T_{c} / \rho g \rightarrow \infty$ so that the forth variable of $f$ also drops. We thus conclude that

$$
\frac{\hbar D N}{\rho g}=f\left(\frac{k_{B} T}{\rho g}\right) .
$$

In Fig 5 we show the graph of $f$ as obtained by our classical field model collecting all the simulation results of Fig 4 and Fig, 3 . We have used a log-log scale in Fig, 5 to reveal that the function $f$ is approximately a power law in the considered range of $k_{B} T / \rho g$.

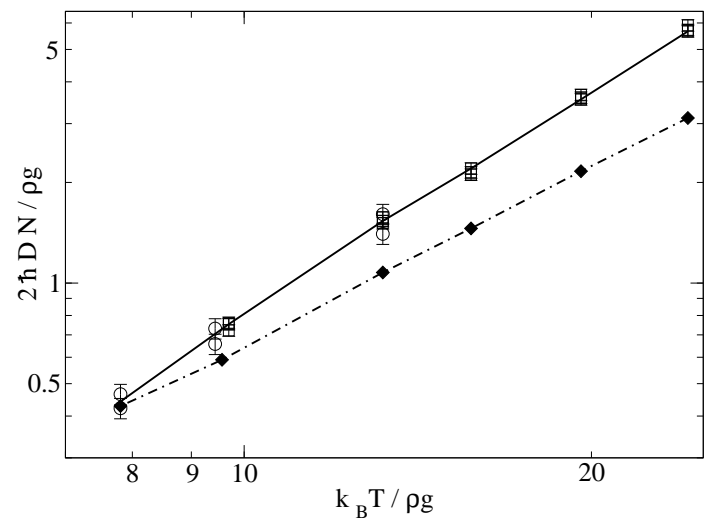

FIG. 5: Universal curve for the rescaled phase diffusion coefficient as a function of $k_{B} T / \rho g$ in $\log$-log scale. Symbols with error bars: Simulation results. Circles: Results of Fig 3 . Squares: Results of Fig 4 The solid line connects the points with the largest value of $N$ of Fig 4 , plus the average of the two points of Fig 3 with the lowest temperature. Dasheddotted line with filled diamonds: $D^{\text {approx }}$ from the projected Gaussian approach. The $\Gamma_{\mathbf{k}}$ for the projected Gaussian approach are calculated on the same discrete grids used in the simulation points connected by the solid line.

\section{PROJECTED GAUSSIAN APPROXIMATION}

In this section we propose an approximate analytical formula for the phase diffusion coefficient that gives some physical insight and can be extended to the quantum field case.

\section{A. Classical field}

We wish to calculate the integral of a correlation function $\mathcal{C}(t)=\langle A(t) A(0)\rangle-\langle A(t)\rangle\langle A(0)\rangle$ of an observable $A$ of the form

$$
A=\sum_{\mathbf{k} \neq \mathbf{0}} A_{\mathbf{k}}\left|b_{\mathbf{k}}\right|^{2}
$$

where $b_{\mathbf{k}}$ are the amplitudes of the field over the Bogoliubov modes. We thus introduce the $\mathcal{M} \times \mathcal{M}$ covariance 
matrix $Q$ with matrix elements

$$
Q_{\mathbf{k}, \mathbf{k}^{\prime}}(t)=\left\langle\delta n_{\mathbf{k}}(t) \delta n_{\mathbf{k}^{\prime}}(0)\right\rangle
$$

where $\delta n_{\mathbf{k}}=n_{\mathbf{k}}-\bar{n}_{\mathbf{k}}$ is the fluctuation of the occupation number of the corresponding Bogoliubov mode and $\mathcal{M}=$ $V /\left(l_{1} l_{2} l_{3}\right)-1$ is the number of Bogoliubov modes. One thus has

$$
\mathcal{C}(t)=\vec{A} \cdot Q(t) \vec{A}
$$

where $\vec{A}$ is the vector of components $A_{\mathbf{k}}$. Since the system is described in this section by the microcanonical ensemble for the Bogoliubov Hamiltonian, the matrix $Q$ obeys the relation

$$
\vec{\epsilon} \cdot Q={ }^{t} \overrightarrow{0} \text { and } Q \vec{\epsilon}=\overrightarrow{0},
$$

where the vector $\vec{\epsilon}$ collects the Bogoliubov energies.

By using the microcanonical classical averages [40] one directly accesses the $t=0$ value of the matrix $Q$,

$$
\begin{array}{ll}
\text { for } \mathbf{k} \neq \mathbf{k}^{\prime}: & Q_{\mathbf{k}, \mathbf{k}^{\prime}}(0)=-\frac{\bar{n}_{\mathbf{k}} \bar{n}_{\mathbf{k}^{\prime}}}{\mathcal{M}+1} \\
\text { for } \mathbf{k}=\mathbf{k}^{\prime}: & Q_{\mathbf{k}, \mathbf{k}}(0)=\bar{n}_{\mathbf{k}}^{2} \frac{\mathcal{M}-1}{\mathcal{M}+1} .
\end{array}
$$

Remarkably we can express this result in terms of the result one would have in the canonical ensemble with average energy equal to the microcanonical energy, adding a projector which suppresses energy fluctuations:

$$
Q(0)=\frac{\mathcal{M}}{\mathcal{M}+1} P^{\dagger} Q^{\text {Gauss }}(0) P
$$

with

$$
P_{\mathbf{k}, \mathbf{k}^{\prime}}=\delta_{\mathbf{k}, \mathbf{k}^{\prime}}-\epsilon_{\mathbf{k}} \alpha_{\mathbf{k}^{\prime}}
$$

The vector $\vec{\alpha}$ is adjoint to the vector $\vec{\epsilon}$ so that $P \vec{\epsilon}=\overrightarrow{0}$. Its components are given by

$$
\alpha_{\mathbf{k}}=\frac{1}{\mathcal{M} \epsilon_{\mathbf{k}}}
$$

and $Q^{\text {Gauss }}$ is the value of the covariance matrix in the canonical ensemble

$$
Q_{\mathbf{k}, \mathbf{k}^{\prime}}^{\mathrm{Gauss}}(0)=\delta_{\mathbf{k}, \mathbf{k}^{\prime}} \bar{n}_{\mathbf{k}}^{2}
$$

The apex "Gauss" reminds the fact that the $b_{\mathbf{k}}$ have a Gaussian probability distribution in the canonical ensemble, contrarily to the case of the microcanonical ensemble.

For the $t=0$ value of the phase derivative correlation function one then obtains

$$
\mathcal{C}(0)=\frac{\mathcal{M}}{\mathcal{M}+1}\left[\sum_{\mathbf{k} \neq \mathbf{0}} A_{\mathbf{k}}^{2} \bar{n}_{k}^{2}-\frac{1}{\mathcal{M}}\left(\sum_{\mathbf{k} \neq \mathbf{0}} A_{\mathbf{k}} \bar{n}_{k}\right)^{2}\right]
$$

with

$$
A_{\mathbf{k}}=-\frac{g}{V}\left(\tilde{U}_{k}+\tilde{V}_{k}\right)^{2}
$$

We verified that (33), represented as a dashed line in Fig,2, is in agreement with the numerical simulation as one enters the Bogoliubov limit (17). Note that within the Bogoliubov aproximation $b_{\mathbf{k}}(t) \simeq b_{\mathbf{k}}(0) e^{-i \tilde{\epsilon}_{k} t \hbar}$, the phase derivative correlation function remains equal to its $t=0$ value (33) at all times. This is in clear disagreement with the numerical simulation, and it would lead to a ballistic spreading of the condensate phase.

Our approximate treatment consists in extending the relation (29) at positive times, using the fact that in a Gaussian theory one would have

$$
Q_{\mathbf{k}, \mathbf{k}^{\prime}}^{\text {Gauss }}(t)=Q_{\mathbf{k}, \mathbf{k}^{\prime}}^{\text {Gauss }}(0) e^{-\Gamma_{\mathbf{k}} t} .
$$

One indeed assumes in the Gaussian model

$$
\left|\left\langle b_{\mathbf{k}}^{*}(t) b_{\mathbf{k}^{\prime}}(0)\right\rangle_{\text {Gauss }}\right|=\delta_{\mathbf{k}, \mathbf{k}^{\prime}} \bar{n}_{k} e^{-\Gamma_{\mathbf{k}} t / 2}
$$

and one uses Wick theorem to obtain (35). Physically equation (36) describes Landau Beliaev processes that decorrelate the $b_{\mathbf{k}}$. It can be derived for example with a master equation approach as done in an appendix of [13].

For the phase derivative correlation function one then obtains the approximate expression

$$
\begin{aligned}
\mathcal{C}^{\operatorname{approx}}(t) & =\frac{\mathcal{M}}{\mathcal{M}+1}\left[\sum_{\mathbf{k} \neq \mathbf{0}} A_{\mathbf{k}}^{2} \bar{n}_{k}^{2} e^{-\Gamma_{\mathbf{k}} t}\right. \\
& -\frac{2}{\mathcal{M}}\left(\sum_{\mathbf{k} \neq \mathbf{0}} A_{\mathbf{k}} \bar{n}_{k}\right)\left(\sum_{\mathbf{k}^{\prime} \neq \mathbf{0}} A_{\mathbf{k}^{\prime}} \bar{n}_{k^{\prime}} e^{-\Gamma_{\mathbf{k}^{\prime}} t}\right) \\
& \left.+\frac{1}{\mathcal{M}^{2}}\left(\sum_{\mathbf{q} \neq \mathbf{0}} e^{-\Gamma_{q} t}\right)\left(\sum_{\mathbf{k} \neq \mathbf{0}} A_{\mathbf{k}} \bar{n}_{k}\right)^{2}\right] .
\end{aligned}
$$

We represent (37) as a dashed-dotted line in Fig 2, The resulting approximation on the diffusion coefficient is obtained by integration

$$
D^{\text {approx }}=\int_{0}^{+\infty} \mathcal{C}^{\operatorname{approx}}(t) d t .
$$

In Fig 5 we compare the approximation (38) (diamonds linked by a dashed-dotted line) to the numerical simulation results. The agreement is acceptable in the considered range of $k_{B} T / \rho g$. The Landau-Beliaev damping rates $\Gamma_{\mathbf{k}}$ are calculated on the same discrete grid as the simulation points as explained in [41].

\section{B. Quantum field}

In this subsection we extend the approximate formula for the phase diffusion coefficient to the quantum case. The Bogoliubov amplitudes and occupation numbers are now operators $\hat{b}_{\mathbf{k}}, \hat{n}_{\mathbf{k}}$. As in the classical case we introduce the covariance matrix of the Bogoliubov occupation numbers

$$
Q_{\mathbf{k}, \mathbf{k}^{\prime}}(t)=\left\langle\delta \hat{n}_{\mathbf{k}}(t) \delta \hat{n}_{\mathbf{k}^{\prime}}(0)\right\rangle
$$


To obtain the $t=0$ value of $Q$ we need to compute quantum averages in the microcanonical ensemble. To this end we use a result derived in [13] giving the first deviation between the microcanonical expectation value $\langle O\rangle$ and the canonical one $\langle O\rangle_{\text {can }}(T)$ in the limit of a large system for an arbitrary observable $O$ :

$$
\langle O\rangle-\langle O\rangle_{\mathrm{can}}(T)=-\frac{1}{2} k_{B} T^{2} \frac{d}{d T}\left(\frac{d\langle O\rangle_{\mathrm{can}} / d T}{d\langle H\rangle_{\mathrm{can}} / d T}\right)+\ldots
$$

where the canonical temperature $T$ is such that the mean energy $\langle H\rangle_{\text {can }}$ in the canonical ensemble is equal to the microcanonical energy $E$. finds

For $\mathbf{k} \neq \mathbf{k}^{\prime}$, using $d \bar{n}_{k} / d T=\epsilon_{k} \bar{n}_{k}\left(\bar{n}_{k}+1\right) / k_{B} T^{2}$, one

$$
Q_{\mathbf{k}, \mathbf{k}^{\prime}}(0) \simeq-\frac{\epsilon_{k} \epsilon_{k^{\prime}} \bar{n}_{k}\left(\bar{n}_{k}+1\right) \bar{n}_{k^{\prime}}\left(\bar{n}_{k^{\prime}}+1\right)}{\sum_{\mathbf{q} \neq \mathbf{0}} \epsilon_{q}^{2} \bar{n}_{q}\left(\bar{n}_{q}+1\right)} .
$$

This scales as $1 / \mathcal{M}$ in the thermodynamic limit. Since the number of off-diagonal terms of $Q$ in (25) is about $\mathcal{M}$ times larger than the number of diagonal terms of $Q$, we have for consistency to calculate the diagnal terms of $Q$ up to order $1 / \mathcal{M}^{0}$, that is the deviation from the canonical value $\bar{n}_{k}\left(\bar{n}_{k}+1\right)$ is not required. To exactly obtain the energy conservation (26), it is however convenient to include, rather than the exact deviation between the canonical and microcanonical values, an ad hoc approximate correction of order $1 / \mathcal{M}$ :

$$
Q_{\mathbf{k}, \mathbf{k}} \simeq \bar{n}_{k}\left(\bar{n}_{k}+1\right)-\frac{\epsilon_{k}^{2} \bar{n}_{k}^{2}\left(\bar{n}_{k}+1\right)^{2}}{\sum_{\mathbf{q} \neq \mathbf{0}} \epsilon_{q}^{2} \bar{n}_{q}\left(\bar{n}_{q}+1\right)}
$$

In this way, we recover the structure of (29), where the $t=0$ value of $Q$ is deduced from the one in the canonical ensemble,

$$
Q_{\mathbf{k}, \mathbf{k}^{\prime}}^{\mathrm{Gauss}}(t=0)=\bar{n}_{k}\left(\bar{n}_{k}+1\right) \delta_{\mathbf{k}, \mathbf{k}^{\prime}}
$$

by the action of a projector $P$,

$$
Q(t=0) \simeq P^{\dagger} Q^{\text {Gauss }}(t=0) P .
$$

The projector $P$ still involves the dyadic structure (30), with a new expression for the vector $\vec{\alpha}$ :

$$
\alpha_{\mathbf{k}}=\frac{\epsilon_{k} \bar{n}_{k}\left(\bar{n}_{k}+1\right)}{\sum_{\mathbf{q} \neq \mathbf{0}} \epsilon_{q}^{2} \bar{n}_{q}\left(\bar{n}_{q}+1\right)} .
$$

As a check, one can apply the classical field limit to the above quantum expressions. One recovers (29), apart from the global factor $\mathcal{M} /(\mathcal{M}+1)$, whose deviation from unity gives rise to terms beyond the accuracy of the present calculation.

At positive times, our quantum projected Gaussian approximation assumes that (44) still holds,

$$
Q^{\text {approx }}(t) \simeq P^{\dagger} Q^{\text {Gauss }}(t) P
$$

with the Gaussian covariance matrix

$$
Q_{\mathbf{k}, \mathbf{k}^{\prime}}^{\text {Gaass }}(t)=\delta_{\mathbf{k}, \mathbf{k}^{\prime}} \bar{n}_{k}\left(\bar{n}_{k}+1\right) e^{-\Gamma_{\mathbf{k}} t}
$$

where the Landau-Beliaev damping rate $\Gamma_{\mathbf{k}}$ is now the usual one, that is for the quantum field theory. From (25) one obtains an approximate expression for the phase derivative correlation function, and from (38) an approximate expression for the quantum field phase diffusion coefficient [42]:

$$
D^{\text {approx }}=\sum_{k \neq \mathbf{0}}\left[(P \vec{A})_{\mathbf{k}}\right]^{2} \frac{\bar{n}_{k}\left(\bar{n}_{k}+1\right)}{\Gamma_{\mathbf{k}}}
$$

where the projection of the vector $\vec{A}$ was introduced:

$$
\begin{aligned}
& (P \vec{A})_{\mathbf{k}}=-\frac{g}{V} \times \\
& {\left[\left(U_{k}+V_{k}\right)^{2}-\epsilon_{k} \frac{\sum_{\mathbf{q} \neq \mathbf{0}} \epsilon_{q}\left(U_{q}+V_{q}\right)^{2} \bar{n}_{q}\left(\bar{n}_{q}+1\right)}{\sum_{\mathbf{q} \neq \mathbf{0}} \epsilon_{q}^{2} \bar{n}_{q}\left(\bar{n}_{q}+1\right)}\right] .}
\end{aligned}
$$

In this expression, the modes amplitudes $U_{k}, V_{k}$ and energy $\epsilon_{k}$ have the usual expressions of the quantum field Bogoliubov theory,

$$
\begin{aligned}
U_{k}+V_{k} & =\frac{1}{U_{k}-V_{k}}=\left(\frac{\hbar^{2} k^{2} / 2 m}{2 \rho g+\hbar^{2} k^{2} / 2 m}\right)^{1 / 4} \\
\epsilon_{k} & =\left[\frac{\hbar^{2} k^{2}}{2 m}\left(2 \rho g+\frac{\hbar^{2} k^{2}}{2 m}\right)\right]^{1 / 2}
\end{aligned}
$$

In appendix $\mathrm{A}$ we give the explicit expression of the Landau-Beliaev damping rates $\Gamma_{k}$ and the approximate phase diffusion coefficient in the thermodynamical limit. The existence of such a limit is due to the fact that none of the momentum integrals involved are infrared divergent, keeping in mind that $\left(U_{k}+V_{k}\right)^{2}, \epsilon_{k}$ and $\Gamma_{k}$ vanish linearly with $k$ [33], while $\bar{n}_{k}\left(\bar{n}_{k}+1\right)$ diverges as $1 / k^{2}$. As expected from the analysis of the previous section, the scaled diffusion coefficient $\hbar D^{\text {approx }} N / \rho g$ is a function of $k_{B} T / \rho g$ only, see (A16).

In Fig 6 we show the values of $D^{\text {approx }}$ in the thermodynamic limit for the same values of $k_{B} T / \rho g$ as in Fig 5. To see the effect of an energy truncation at $k_{B} T$, we also show the values of $D^{\text {approx }}$ obtained by introducing an energy cut-off $\epsilon_{q}<k_{B} T$ in (A16) and the same cut-off $\epsilon_{k}<k_{B} T$ in the integrals (A1A9) giving the damping rate $\Gamma_{q}$. As expected, the resulting values of $D^{\text {approx }}$ are close to the values of $D^{\text {approx }}$ obtained for the classical field model in Fig [5. and that we have reported in Fig 6 for comparison. We conclude that the diffusion coefficient is indeed affected by an energy cut-off, and the coefficient obtained in the classical field simulations with an energy cut-off $k_{B} T$ might differ quantitatively from the real one by a factor of about two.

\section{CONCLUSION}

Using a classical field model, we have shown that the phase of a Bose-Einstein condensate undergoes true diffusion in time, when the gas is initially prepared in the mi- 


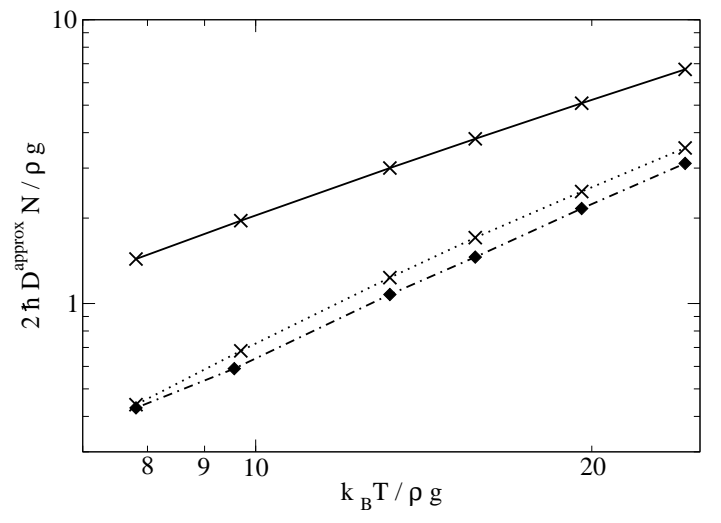

FIG. 6: Approximate phase diffusion coefficient in the thermodynamic limit as a function of $k_{B} T / \rho g$ in log-log scale. Solid line with crosses: $D^{\text {approx }}$ for the quantum field from Eq. A16). Dotted line with crosses: Effect of an energy cutoff equal to $k_{B} T$ in Eq. A16). Dashed-dotted line with filled diamonds: $D^{\text {approx }}$ for the classical field from Eq. (38) (same data as in Fig [5].

crocanonical ensemble. Parametrizing the microcanonical energy $E$ by the temperature $T$ of the canonical ensemble with average energy $E$, we could show that the rescaled diffusion coefficient $\hbar D N / \rho g$, where $N$ is the fixed number of particles and $\rho g$ is the Gross-Pitaevskii chemical potential, is a function of a single variable $k_{B} T / \rho g$ in the double thermodynamic and Bogoliubov limit.

We have derived an approximate formula for the diffusion coefficient, in fair agreement with the classical field simulations. We could generalize the approximate formula to the quantum field case, show that it also admits a thermodynamic limit and that it satisfies the scaling property found for the classical field. We have used the quantum approximate formula to evaluate the effect of an energy cut-off, not required in the quantum theory and unavoidable in the classical field model.

The perspective of using the condensate phase spreading to experimentally distinguish among different statistical ensembles is fascinating, although the measurement of the intrinsic phase diffusion of a Bose-Einstein condensate discussed here remains a challenge and will be the subject of further investigations.

\section{ACKNOWLEDGMENTS}

The authors would like to acknowledge E. Witkowska for her initial contribution to the project, K. Mølmer for hospitality and useful discussions and F. Hulin-Hubard for valuable help with computers. A.S. acknowledges stimulating discussions with W. Phillips, K. Rzążewski, M. Gajda and M. Oberthaler. Our groups are members of IFRAF.

\section{APPENDIX A: LANDAU-BELIAEV DAMPING RATES AND APPROXIMATE PHASE DIFFUSION COEFFICIENT IN THE THERMODYNAMIC LIMIT}

We start with the Landau damping rate of a Bogoliubov mode of wave vector $\mathbf{q}$ as given in 13 .

$$
\Gamma_{q}^{L}=\frac{g^{2} \rho}{\pi^{2} \hbar} \int d^{3} k L_{k, k^{\prime}}^{2}\left(\bar{n}_{k}-\bar{n}_{k^{\prime}}\right) \delta\left(\epsilon_{q}+\epsilon_{k}-\epsilon_{k^{\prime}}\right)
$$

with

$$
L_{k, k^{\prime}}=U_{q} V_{k} U_{k^{\prime}}+\left(U_{q}+V_{q}\right)\left(U_{k} U_{k^{\prime}}+V_{k} V_{k^{\prime}}\right)+V_{q} U_{k} V_{k^{\prime}} .
$$

The mode of wave vector $\mathbf{q}$ scatters an excitation of wave vector $\mathbf{k}$ giving rise to an excitation of wave vector $\mathbf{k}^{\prime}$. The final mode has to satisfy momentum conservation so that $\mathbf{k}^{\prime}=\mathbf{k}+\mathbf{q}$. Energy conservation $\epsilon_{q}+\epsilon_{k}=\epsilon_{k^{\prime}}$ is ensured by the delta distribution in (A1). In the integral over $\mathbf{k}$ we use spherical coordinates of axis $\mathbf{q}, \theta$ being the polar angle. We introduce the momentum $\check{q}$ scaled by the inverse of the healing length $\xi$ and the mode energy $\check{\epsilon}_{q}$ scaled by the Gross-Pitaevskii chemical potential $\rho g$ :

$$
\begin{gathered}
\check{q}=q\left(\frac{\hbar^{2}}{2 m \rho g}\right)^{1 / 2}=q \xi, \\
\check{\epsilon}_{q}=\frac{\epsilon_{q}}{\rho g}=\left[\check{q}^{2}\left(\check{q}^{2}+2\right)\right]^{1 / 2} .
\end{gathered}
$$

As a consequence, the mean occupation number $\bar{n}_{q}$ is a function of $\check{q}$ and of the ratio $k_{B} T / \rho g$ only, and the mode amplitudes $U_{q}, V_{q}$ are functions of $\tilde{q}$ only. Introducing the notation $u=\cos \theta$, one has

$$
\delta\left(\check{\epsilon}_{q}+\check{\epsilon}_{k}-\check{\epsilon}_{k^{\prime}}\right)=\delta\left(u-u_{0}^{L}\right) \frac{\check{\epsilon}_{q}+\check{\epsilon}_{k}}{2 \check{k} \check{q}\left[1+\left(\check{\epsilon}_{q}+\check{\epsilon}_{k}\right)^{2}\right]^{1 / 2}}
$$

where

$$
u_{0}^{L}=\frac{\left[1+\left(\check{\epsilon}_{q}+\check{\epsilon}_{k}\right)^{2}\right]^{1 / 2}-\left(1+\check{q}^{2}+\check{k}^{2}\right)}{2 \check{k} \check{q}} .
$$

One can show that $u_{0}^{L}$ is in between -1 and 1 for all values of $\check{k}$ and $\check{q}$, so that the angular integration is straightforward and leads to

$$
\Gamma_{q}^{L}=\frac{g}{\pi \hbar \xi^{3}} \int_{0}^{+\infty} d \check{k} L_{k, k^{\prime}}^{2} \frac{\check{k}^{\prime}\left(\check{\epsilon}_{k}+\check{\epsilon}_{q}\right)\left(\bar{n}_{k}-\bar{n}_{k^{\prime}}\right)}{\check{q}\left[1+\left(\check{\epsilon}_{q}+\check{\epsilon}_{k}\right)^{2}\right]^{1 / 2}}
$$

with

$$
1+\check{k}^{\prime 2}=\left[1+\left(\check{\epsilon}_{q}+\check{\epsilon}_{k}\right)^{2}\right]^{1 / 2} .
$$

A similar procedure may be applied to the Beliaev damping rate for the mode q. From [13] one has

$$
\Gamma_{q}^{B}=\frac{g^{2} \rho}{2 \pi^{2} \hbar} \int d^{3} k B_{k, k^{\prime}}^{2}\left(1+\bar{n}_{k}+\bar{n}_{k^{\prime}}\right) \delta\left(\epsilon_{k}+\epsilon_{k^{\prime}}-\epsilon_{q}\right)
$$


with

$$
B_{k, k^{\prime}}=U_{q} U_{k} U_{k^{\prime}}+\left(U_{q}+V_{q}\right)\left(V_{k} U_{k^{\prime}}+U_{k} V_{k^{\prime}}\right)+V_{q} V_{k} V_{k^{\prime}} .
$$

Here the mode of wave vector $\mathbf{q}$ decays into an excitation of wave vector $\mathbf{k}$ and an excitation of wave vector $\mathbf{k}^{\prime}$. Momentum conservation imposes $\mathbf{k}^{\prime}=\mathbf{q}-\mathbf{k}$. Energy conservation $\epsilon_{k^{\prime}}=\epsilon_{q}-\epsilon_{k}$ is ensured by the delta distribution in (A9), and clearly imposes $k<q$. With the same scaled variables and spherical coordinates as above, one obtains

$$
\delta\left(\check{\epsilon}_{k}+\check{\epsilon}_{k^{\prime}}-\check{\epsilon}_{q}\right)=\delta\left(u-u_{0}^{B}\right) \frac{\check{\epsilon}_{q}-\check{\epsilon}_{k}}{2 \check{k} \check{q}\left[1+\left(\check{\epsilon}_{q}-\check{\epsilon}_{k}\right)^{2}\right]^{1 / 2}}
$$

where

$$
u_{0}^{B}=\frac{1+\check{q}^{2}+\check{k}^{2}-\left[1+\left(\check{\epsilon}_{q}-\check{\epsilon}_{k}\right)^{2}\right]^{1 / 2}}{2 \check{k} \check{q}} .
$$

One can show that $u_{0}^{B}$ is in between -1 and 1 , whatever the values of $\check{q}$ and $\check{k}<\check{q}$, so that angular integration is straightforward and gives

$$
\Gamma_{q}^{B}=\frac{g}{2 \pi \hbar \xi^{3}} \int_{0}^{\check{q}} d \check{k} B_{k, k^{\prime}}^{2} \frac{\check{k}_{(}\left(\check{\epsilon}_{q}-\check{\epsilon}_{k}\right)\left(1+\bar{n}_{k}+\bar{n}_{k^{\prime}}\right)}{\check{q}\left[1+\left(\check{\epsilon}_{q}-\check{\epsilon}_{k}\right)^{2}\right]^{1 / 2}}
$$

with

$$
1+\check{k}^{\prime 2}=\left[1+\left(\check{\epsilon}_{q}-\check{\epsilon}_{k}\right)^{2}\right]^{1 / 2}
$$

Finally we introduce the rescaled total damping rate,

$$
\check{\Gamma}_{q}=\frac{2 \pi^{2} \hbar \xi^{3}}{g}\left(\Gamma_{q}^{L}+\Gamma_{q}^{B}\right)
$$

a dimensionless function of $k_{B} T / \rho g$ only. From (48) one then obtains in the thermodynamic limit an approximate expression for the phase diffusion coefficient depending only on $k_{B} T / \rho g$,

$$
\frac{\hbar D^{\text {approx }} N}{\rho g}=\int_{0}^{+\infty} d \check{q} \check{q}^{2}\left(\mathcal{A}_{q}^{P}\right)^{2} \frac{\bar{n}_{q}\left(1+\bar{n}_{q}\right)}{\check{\Gamma}_{q}}
$$

with

$$
\mathcal{A}_{q}^{P}=\left(U_{q}+V_{q}\right)^{2}-\check{\epsilon}_{q} \frac{\int_{0}^{+\infty} d \check{k} \check{k}^{2} \check{\epsilon}_{k}\left(U_{k}+V_{k}\right)^{2} \bar{n}_{k}\left(\bar{n}_{k}+1\right)}{\int_{0}^{+\infty} d \check{k}^{2} \check{k}^{2} \check{\epsilon}_{k}^{2} \bar{n}_{k}\left(\bar{n}_{k}+1\right)} .
$$

[1] K. Bongs, K. Sengstock, Reports on Progress in Physics 67, 907-963 (2004); T. Schumm, S. Hofferberth, L.M. Anderson, S. Wildermuth, S. Groth, I. Bar-Joseph, J. Schmiedmayer, P. Krüger, Nature Physics 1, 57 (2005); P. Treutlein, P. Hommelhoff, T. Steinmetz, T. W. Hänsch, J. Reichel, Phys. Rev. Lett. 92, 203005 (2004); O. Mandel, M. Greiner, A. Widera, T. Rom, T.W. Hänsch, I. Bloch, Nature 425, 937 (2003); A. Micheli, D. Jaksch, I. Cirac, P. Zoller, Phys. Rev. A 67, 013607 (2003).

[2] E.M. Wright, D.F. Walls, J.C. Garrison, Phys. Rev. Lett. 77, 2158 (1996); J. Javanainen, M. Wilkens, Phys. Rev. Lett. 78, 4675 (1997) [see also the comment by A. Leggett, F. Sols, Phys. Rev. Lett. 81, 1344 (1998), and the related answer by J. Javanainen, M. Wilkens, Phys. Rev. Lett. 81, 1345 (1998)]; M. Lewenstein, Li You, Phys. Rev. Lett. 77, 3489 (1997); Y. Castin, J. Dalibard, Phys. Rev. A 55, 4330 (1997); P. Villain, M. Lewenstein, R. Dum, Y. Castin, Li You, A. Imamoglu, T.A.B. Kennedy, Journal of Modern Optics, 44 1775-1799 (1997); A. Sinatra, Y. Castin, Eur. Phys. J. D 4, 247-260 (1998); A. Sinatra and Y. Castin, Eur. Phys. J. D 8, 319 (2000).

[3] M.R. Andrews, C.G. Townsend, H.J. Miesner, D.S. Durfee, D.M. Kurn, W. Ketterle, Science 275, 637 (1997).

[4] D.S. Hall, M.R. Matthews, C.E. Wieman, and E.A. Cornell, Phys. Rev. Lett. 81, 1543 (1998).

[5] C. Orzel, A.K. Tuchman, M.L. Fenselau, M. Yasuda, M. Kasevich, Science 291, 2386 (2001); M. Greiner, O. Mandel, T.W. Hansch, I. Bloch, Nature 419, 51 (2002); Y. Shin, M. Saba, T.A. Pasquini, W. Ketterle, D.E. Pritchard, A.E. Leanhardt, Phys. Rev. Lett. 92, 050405
(2004).

[6] G.-B. Jo, Y. Shin, S. Will, T. A. Pasquini, M. Saba, W. Ketterle, D. E. Pritchard, M. Vengalattore, M. Prentis, Phys. Rev. Lett. 98, 030407 (2007); G.-B. Jo, J.-H. Choi, C. A. Christensen, Y.-R. Lee, T. A. Pasquini, W. Ketterle, D. E. Pritchard, Phys. Rev. Lett. 99, 240406 (2007).

[7] A. Widera, S. Trotzky, P. Cheinet, S. Fölling, F. Gerbier, I. Bloch, V. Gritsev, M. D. Lukin, and E. Demler, Phys. Rev. Lett. 100, 140401 (2008).

[8] S. Hofferberth, I. Lesanovsky, B. Ficher, T. Shumm, J. Schmiedmayer, Nature 449, 324 (2007).

[9] A. A. Burkov, M. D. Lukin, E. Demler, Phys. Rev. Lett. 98, 200404 (2007).

[10] L. Pitaevskii, S. Stringari, Phys. Rev. Lett. 87, 180402 (2001).

[11] R. Gati, B. Hemmerling, J. Folling, M. Albiez, M. K. Oberthaler, Phys. Rev. Lett. 96130404 (2006)

[12] F. Sols, Physica B 194-196, 1389 (1994)

[13] A. Sinatra, Y. Castin, E. Witkovska, Phys. Rev. A 75, 0033616 (2007).

[14] A.B. Kuklov, J.L. Birman, Phys. Rev. A 63, 013609 (2001).

[15] D. Jaksch, C. W. Gardiner, K. M. Gheri, P. Zoller, Phys. Rev. A 58, 1450 (1998).

[16] R. Graham, Phys. Rev. Lett. 81, 5262 (1998).

[17] R. Graham, Phys. Rev. A 62, 023609 (2000).

[18] R. Graham, Journal of Mod. Opt. 47, 2615 (2000).

[19] Yu. Kagan, B. V. Svistunov, and G. V. Shlyapnikov, Sov. Phys. JETP 75, 387 (1992); Yu. Kagan and B. Svistunov, Phys. Rev. Lett. 793331 (1997). 
[20] K. Damle, S. N. Majumdar and S. Sachdev, Phys. Rev. A 54, 5037 (1996).

[21] M. J. Steel, M. K. Olsen, L. I. Plimak, P. D. Drummond, S. M. Tan, M. J. Collett, D. F. Walls, R. Graham, Phys. Rev. A 58, 4824 (1998).

[22] K. Góral, M. Gajda, K. Rzążewski, Opt. Express 8, 92 (2001); D. Kadio, M. Gajda and K. Rza̧żewski, Phys. Rev. A 72, 013607 (2005).

[23] M.J. Davis, S.A. Morgan and K. Burnett, Phys. Rev. Lett. 87, 160402 (2001).

[24] A. Sinatra, C. Lobo, Y. Castin, Phys. Rev. Lett. 87, 210404 (2001).

[25] $\Delta$ stands here for the operator acting on functions on the lattice, such that its eigenvectors are the plane waves $e^{i \mathbf{k} \cdot \mathbf{r}}$ with eigenvalues $-k^{2}$.

[26] We calculate $\operatorname{Var} \varphi(t)$ and its error bars for 5000 time points. These 5000 time points are collected into 100 packets of 50 consecutive points, the $i^{\text {th }}$ packet corresponding to a time interval $\left[t_{i}, t_{i}+\Delta t\right] . \Delta t$ is chosen of the order of the typical correlation time of the condensate phase derivative (see Fig 2). A temporal average of $\operatorname{Var} \varphi$ over each time interval is then calculated. and reported in the figure as a single point at $t_{i}+\Delta t / 2$ with its mean square (time averaged) error bar.

[27] As explained in [13], in (16) we assume a small non condensed fraction and we neglect an oscillatory part. We checked analytically, within the Bogoliubov approach and for an infinite cut-off, that the contribution of the oscillatory part of $\dot{\varphi}$ to the correlation function $\mathcal{C}(t)$ is a rapidly decreasing function over the time scale $\hbar / \rho g$.

[28] In our classical field model, the field amplitudes on the Bogoliubov modes are given by $b_{\mathbf{k}}(t)=$ $d V \sum_{\mathbf{r}} \tilde{U}_{k} \frac{e^{-i \mathbf{k} \cdot \mathbf{r}}}{\sqrt{V}} e^{-i \theta(t)} \psi_{\perp}(\mathbf{r}, t)+\tilde{V}_{k} \frac{e^{i \mathbf{k} \cdot \mathbf{r}}}{\sqrt{V}} e^{i \theta(t)} \psi_{\perp}^{*}(\mathbf{r}, t)$ where $\psi_{\perp}$ is the component of $\psi$ orthogonal to the condensate mode and the real amplitudes $\tilde{U}_{k}, \tilde{V}_{k}$, normalized as $\tilde{U}_{k}^{2}-\tilde{V}_{k}^{2}=1$, are given by the usual Bogoliubov theory, here with the modified dispersion relation $\tilde{U}_{k}+\tilde{V}_{k}=\left[\tilde{E}_{k} /\left(\tilde{E}_{k}+2 \rho g\right)\right]^{1 / 4}$.
[29] Y. Castin, R. Dum, Phys. Rev. A 57, 3008 (1998).

[30] Taking this limit in its true mathematical sense, we eventually reach a discrete regime where the Bogoliubov eigenstates do not form a quasi-continuum, since the energy spacings between the Bogoliubov energy levels are fixed in the Bogoliubov limit whereas the coupling amplitudes among them vanish in this limit. The concept of Beliaev-Landau damping rates may be not appropriate in this discrete regime. To be more rigorous mathematically one should first take the thermodynamic limit, and then take the Bogoliubov limit (now formulated as $\rho \rightarrow+\infty$ for a fixed $\rho g$ ). Here we have kept the opposite order for pedagogical reasons, the intuitive reasoning in the Bogoliubov limit allowing to infer the proportionality of $D$ with $1 / N$.

[31] Vincent Liu, Phys. Rev. Lett. 79, 4056 (1997).

[32] L.P. Pitaevskii, S. Stringari, Phys. Lett. A 235, 398 (1997).

[33] S. Giorgini, Phys. Rev. A 57, 2949 (1998).

[34] P. Fedichev, G. Shlyapnikov, Phys. Rev. A 58, 3146 (1998).

[35] A. Sinatra, P. Fedichev, Y. Castin, J. Dalibard, G. Shlyapnikov, Phys. Rev. Lett. 82 251-254 (1998).

[36] H. Schmidt, K. Góral, F. Floegel, M. Gajda, K. Rzążewski, J. Opt. B 5, S96 (2003).

[37] M. Brewczyk, P. Borowski, M. Gajda, K. Rzążewski, J. Phys. B 37, 2725 (2004).

[38] C. Lobo, A. Sinatra and Y. Castin, Phys. Rev. Lett. 92, 020403 (2004); N.G. Parker, C.S. Adams, Phys. Rev. Lett. 95, 145301 (2005).

[39] A. A. Norrie, R. J. Ballagh, C. W. Gardiner, Phys. Rev. Lett. 94, 040401 (2005).

[40] S. Aubert, C.S. Lam, J. Math Phys. 44, 6112 (2003).

[41] A. Sinatra, C. Lobo, Y. Castin, J. Phys. B 35, 3599 (2002).

[42] One may note that our approximation for $Q(t)$ is a symmetric matrix, which is not necessarily true for the exact $Q$ at $t>0$. 